\title{
La patologización de la conductaः discursos asistenciales acerca del trastorno disocial ${ }^{1}$
}

\author{
Paloma Garrido Hernández \\ Universitat Rovira i Virgili \\ paloma.gh.48@gmail.com
}

Resumen: En los últimos años, nuestra sociedad ha asistido al crecimiento del número de diagnósticos de diferentes tipos de trastornos de conducta entre la población infantil y adolescente. Desde diferentes disciplinas, principalmente del ámbito de la salud y la educación, diversos autores han investigado las causas de este fenómeno, así como las posibles soluciones. Sin embargo, a menudo estos estudios han dejado de lado el papel de un actor esencial en el proceso terapéutico: el experto. Con la idea de desnaturalizar los discursos a partir de los cuales se patologiza el comportamiento y se prescriben pautas para su transformación, en este trabajo el foco se centrará precisamente en las experiencias y perspectivas de trabajadores del circuito asistencial, buscando analizar sus definiciones de salud y enfermedad, la relación que establecen entre la patología y el contexto cercano y amplio y la identidad que atribuyen al paciente. Para ello, he decidido centrarme en el trastorno disocial, un diagnóstico aplicado a las poblaciones más jóvenes, que se determina a partir de criterios basados en la observación de agentes externos al paciente, no tanto de su propia experiencia subjetiva, y que se ha mostrado profundamente condicionado por el contexto cercano del individuo, revelando su carácter de categoría cultural.

Palabras clave: patologización; educación; infancia; adolescencia; trastorno disocial; trastorno de conducta.

Abstract: In recent years, our society has seen the increase of a number of different kinds of diagnoses of behavioral disorders within the child and teen population. From different areas, mainly within the field of health and education, various authors have investigated the causes of this phenomenon, and its possible solutions. However, more often than not, these studies have

1 Este trabajo resultó de la investigación que realicé como trabajo final de máster, tutorizado por Ángel Martínez Hernáez. 
left on a side the role of the principal actor in the therapeutic process: the expert. With the idea of denaturalize the speeches from which it pathologises the behavior and guidelines prescribed to their transformation, this paper will focus primarily on the experiences and perspectives of workers within the care circuit, seeking to analyse their definitions of health and sickness, the relationship established between pathology and close and broader context and the identity attributed to the patient. To do so, I have decided to focus on disocial disorder, a disorder applied to youth populations, determined through criteria based on the observation of external agents to the patient, if not so much from their own subjective experience, and has shown deeply influenced by the proximity of the individual context, revealing its nature as cultural category.

Keywords: Pathologization; Education; Childhood; Adolescence; Dissocial Disorder; Conduct Disorder.

\section{Introducción}

Good (2003) señalaba la dificultad de superar la convicción de que nuestro sistema de conocimiento se corresponde con el orden natural de la realidad y de que nuestras categorías son "descriptivas", no culturales, construidas y clasificatorias. Sin embargo, desde diferentes ámbitos científicos se reconoce cada vez más el hecho de que determinadas disciplinas se fundamentan en gran medida en juicios y consideraciones éticos, que quedan ocultos tras una declaración de objetividad (Szasz, 1970).

Frente a la concepción biomédica hegemónica, desde la antropología se plantea la enfermedad no solo como una circunstancia biológica (disease), sino también como un conjunto de significados culturales (illness) y de interacciones sociales (sickness) de cuyo estudio es posible desprender los símbolos de la sociedad en la que se ubican (Young, 1982; Kleinman, 1991; Good, 2003; Martínez, 2008). Ya que es necesario incluir en investigación el estudio de las condiciones sociales de producción del conocimiento (Young, 1982), pretendo 
analizar de qué maneras y bajo qué argumentos, líneas discursivas y representaciones se desarrolla el circuito asistencial médico, atendiendo también al lugar que dan al paciente en el mismo.

Considero el estudio del trastorno disocial (TD a partir de ahora) como un caso especialmente interesante en la medida en que aúna, tanto en su definición como en las estrategias a partir de las cuales se aborda, las instituciones médicas y las educativas, ya que, como afirma Gustavo Mariluz (ICP, 2015), la educación no es una institución neutral, un mero agente socializador, sino una forma de construir a los sujetos que forman la sociedad y, por tanto, de construir el mundo.

\subsection{El trastorno disocial en la literatura médica}

El trastorno disocial, aplicado a niños y adolescentes, es el que implica un patrón según el cual el individuo tiene un comportamiento anormal para su edad que le lleva a entrar en conflicto con las normas y con los derechos o necesidades de los demás, lo que implica en ocasiones la comisión de delitos (Romero, 2001; Dios de Vega, 2002; Soutullo y Mardomingo, 2010; Cova et alii, 2012).

A pesar de no haberse dado con una base biológica para este trastorno, sigue presente en diversos textos y estudios la posibilidad de que esta llegue a encontrarse, sea en forma de factores determinantes (Delgado et alii, 1994; Toro, 1999) o de predisposición genética (Moffit, 1993; Delgado et alii, 1994; Toro, 1999; Mobili et alii, 2006; Soutullo et alii, 2010). Dos estudios internacionales que trabajan actualmente en la búsqueda de las bases biológicas del trastorno disocial son Aggressotype $e^{2}$ y Matrics ${ }^{3}$.

Esta atribución del trastorno a la biología, aunque parcial, remite a un modelo explicativo basado en la idea del cuerpo-especie según el cual las circunstancias personales de los individuos están condicionadas en mayor o menor medida por este componente universal. Mediante la inclusión de unas características biológicas determinadas entre los factores condicionantes necesarios, se establece un elemento común a todos los casos definidos como TD que justificaría la clasificación de los denominados síntomas como un trastorno, una categoría médica unificada más allá de las expresiones específicas de cada caso.

\footnotetext{
$2<$ http://www.aggressotype.eu/>

$3<$ http://matrics-project.eu/about/aims-objectives $>$
} 
Con un enfoque menos biologicista, diversos tipos de estudios buscaban establecer conexiones entre el TD y otras circunstancias. En Colombia, Pineda et alii (2001) quisieron corroborar la hipótesis según la cual las conductas antisociales y violentas responderían a un patrón de aprendizaje social, considerando finalmente que las características del fenómeno estudiado apuntan sobre todo a una posible etiología genética del TD. Cova et alii (2012) han señalado una asociación entre un cociente intelectual menor, de discapacidad intelectual, limítrofe y normal lento y las conductas disociales. Por su parte, Ruvalcaba et alii (2012) afirman que una capacidad reducida para empatizar y una inteligencia emocional poco desarrollada predicen la posibilidad de involucrarse en conductas disociales.

Se ha señalado también que la desestructuración familiar, los comportamientos antisociales en este grupo, los diagnósticos de enfermedades mentales en el padre o la madre, la ausencia de figura paterna o la dificultad de compaginar el horario laboral con la educación de los hijos pueden implicar un mayor riesgo para el menor de desarrollar este tipo de conductas (Delgado et alii, 1994; Pineda et alii, 2001; Dios de Vega, 2002; Cova et alii, 2012; Ruvalcaba et alii, 2012; Matalí, 2016; Ferreras, 2016). Así, frente a lo que se percibe como desviación individual o del grupo familiar, las instituciones y los discursos y prácticas expertos prescriben una serie de iniciativas de análisis, remodelación, prevención y control de los estilos de vida (Foucault, 1998; Fassin, 2010).

Únicamente dos estudios hacen referencia a factores estructurales. Pineda et alii (2001) señalan una mayor prevalencia del TD en los estratos socioeconómicos bajos, utilizando la clase social para justificar dinámicas "disfuncionales", reforzando la idea de que a menudo las clases populares son el objetivo principal de los mecanismos de control del biopoder (Foucault, 2007; Caponi, 2012). Por su parte, Matalí (2016) señala que el inicio de la crisis económica de los últimos años ha podido contribuir al incremento de problemas de conducta en los jóvenes y niños. 


\subsection{Psiquiatrización de la diversidad: críticas a la patologización}

El hecho de no haber encontrado correlato orgánico indiscutible para los trastornos descritos en sus manuales diagnósticos implica que el ámbito de la salud mental sea uno de los que más críticas enfrentan dentro del circuito asistencial de la salud en general. Así, desde diferentes disciplinas se ha problematizado el modo en que realidades sociales han sido interpretadas y reconstruidas de manera que han pasado a formar parte de las competencias de la psiquiatría (Vásquez, 2011). Principalmente en el ámbito de la educación, la psicología y la antipsiquiatría, la explicación en la que se incide con mayor ahínco es aquella que refiere a una suerte de cambios en la sociedad y su reflejo en la población infantil y juvenil, que no han tenido correspondencia con las capacidades para gestionarlos desarrolladas por el sistema educativo en concreto y la sociedad adulta en general.

En relación a los cambios sociales que pueden provocar conflictos en y con la infancia, se señala la influencia del mercado y los medios de comunicación como una suerte de nuevos agentes de socialización, como performadores de la conducta, las relaciones sociales y la percepción simbólica. Según esto, las lógicas del deseo y consumo inmediato generarían sujetos en una situación permanente de demanda y consumo compulsivo enfocado a la satisfacción casi inmediata de sus deseos e incapaz de implicarse en procesos a más largo plazo. Además, estas dinámicas tendrían también su incidencia en los proyectos educativos, deseosos de resultados rápidos que permitan continuar, retomar la «normalidad» lo antes posible (Dueñas en ICP, 2015; Medel, 2016; Pajuelo, 2016).

Medel (2016) nos dibuja un momento histórico en el que la experiencia infantil se ha visto afectada por la sustitución de los objetos culturales por los de consumo, con consecuencias en la posición que el niño o niña adopta en el vínculo social. Mientras, los cambios en los modos de información, la nueva accesibilidad del conocimiento a partir de las plataformas virtuales, han alterado una relación que se sostenía en gran parte sobre la asimetría de saber, generando la indiferenciación de ciertas funciones entre adultos e hijos, con la consecuente pérdida de autoridad de las figuras que tradicionalmente habían sido referencia para el infante (Dueñas en ICP, 2015; Medel, 2016). 
Ante estos cambios, se plantea la necesidad de modificar las estrategias seguidas hasta el momento, señalando la posibilidad de que los manuales y métodos utilizados hasta ahora para tratar las cuestiones relativas al comportamiento infantil hayan quedado desfasados, inadecuados para la situación social actual, quedando libre un espacio que los laboratorios farmacéuticos han aprovechado para promover la biomedicalización de las niñas y los niños (Dueñas en ICP, 2015; Medel, 2016; Font y Vidal, 2016; Guijarro, 2016; Herrera, 2016; Pajuelo, 2016), reduciendo problemáticas sociales y familiares profundamente complejas a supuestas deficiencias o trastornos biológicos propios del individuo concreto (Kleinman, 1991; Vásquez, 2011).

Este uso por parte de la psiquiatría de la enfermedad como explicación de la infelicidad y el conflicto humano ha dado lugar a una suerte de «cultura de la terapia» en la que los problemas sociales son patologizados en la medida en que se expresan en personas individuales (González et alii, 2007). Además, esta medicalización genera etiquetas que actúan casi como un alias, atravesando la percepción del medio social y justificando la entrada en escena de la mano médica (Dueñas, 2007; Medel, 2016). Este etiquetamiento puede tener relación con los dispositivos de disciplinamiento de los sujetos, tal y como describió Foucault (2007), enfocados a la producción de subjetividad y al control de la conducta de los sujetos. Se trataría de un poder disciplinar con función normalizadora, individualizadora y centrada en el cuerpo, enfocado a transformar al sujeto a partir del ideal preestablecido mediante elementos que fuerzan el cambio, rozando el autoritarismo (Dueñas, 2007; Medel, 2016).

La constante aparición de categorías diagnósticas disfrazadas de sintomatologías nuevas, que abarcan cada vez aspectos más sutiles de la conducta y la vida cotidiana, ha llevado al planteamiento de la existencia de modas respecto a los trastornos (Szasz, 1970; Martínez, 2016), que, aunque podrían responder a los cambios de la sociedad en las que nacen, parecen formar parte de un proceso de patologización creciente. Rocas (ICP, 2015a) señala la "prevención temprana" como un término que esconde la patologización de gran variedad de tipos de sufrimiento que ella reivindica como legítimos y propios de la vida, afirmando que esa supuesta prevención es la que termina generando patologías, ya que interrumpe los procesos elaborativos de la mente. 
El paso de la atención del trastorno orgánico a la incapacidad percibida y al sufrimiento como criterios para seleccionar al individuo "enfermo" se justifica, en definitiva, en términos de conveniencia social, llegándose a llamar enfermedad a todo lo que "permitiera detectar algún signo de disfunción, sobre la base de cualquier tipo de norma” (Szasz, 2008: 55), pero sin conseguir hacerlo mediante la propia observación científica. Aunque la conducta está condicionada por la biología, lo que la determina en el caso del ser humano serían los roles sociales y las reglas que el grupo impone sobre el individuo. En esta dinámica, el concepto de aprendizaje quedaría ligado al de la actuación: aprendemos cómo actuar, qué papel tenemos que desempeñar en cada situación en función de las expectativas sociales. Así, cuando se basa en comportamientos o juicios subjetivos, el calificativo de "enfermedad" sobre una conducta dependerá del punto de vista adoptado (Szasz, 2008).

\section{Acceso al campo}

\subsection{Enfoque}

Gramsci definía la begemonía como una forma de dominación en la cual la coerción y la violencia no desaparecen, sino que coexisten con formas de aceptación del poder y dominación aparentemente voluntarias o consensuadas con los sujetos subalternos. El "sentido común" quedaría construido a partir de los valores de las clases dominantes y se adquiriría y naturalizaría a través de un proceso en el que la educación, la religión y la cultura tienen un papel crucial (Aguirre, 2007). En relación, Foucault buscó mostrar hasta qué punto el poder y la concepción de la verdad están ligados también en los procesos de subjetivación, no solo en el campo político. Para este autor, la verdad se bastaría a sí misma para ser; lo demás serían todo regímenes de verdad, construcciones interesadas y temporales, generalmente unas en lucha con otras para imponerse como hegemónicas (Lorenzini, 2010). En relación con esto, define el biopoder como el poder sobre la vida, la forma de gobierno en la que, mediante el conocimiento y la acción, los intersticios de la vida se erigirían como objeto principal de la política (Foucault, 1998; Fassin, 2010). 
Hoy en día, siguiendo este proceso de hegemonización del orden moral imperante (Fassin, 1999) y de naturalización de las técnicas de sujeción de los cuerpos y control de las poblaciones (Foucault, 1998), en las sociedades modernas se ha incrementado la tendencia a pensar los conflictos vitales, subjetivos y sociales, en términos médicos y psiquiátricos, reclasificándose numerosos hechos como "enfermedades". Así, para cada anomalía, para cada comportamiento interpretado como desviado, se ha ido generando un nuevo cuadro patológico, sin que exista conducta que no sea susceptible de ser cuestionada psiquiátricamente (Szasz, 2008; Caponi, 2012).

Esta categorización de la conducta correspondería con el objetivo de anticipar la emergencia del peligro, principalmente el representado por las clases populares, consideradas potencialmente peligrosas. Respecto a la dimensión definida como preventiva de la "psiquiatrización de lo social", la infancia se presentó como una población especialmente susceptible de ser medicalizada: ante el riesgo de la cronificación o del desarrollo perjudicial de los cuadros patológicos, las niñas y los niños se convirtieron en objetivo de esta disciplina (Foucault, 2007; Caponi, 2012). Donde lo importante es que el individuo no cuestione ni perturbe el tejido social (Szasz, 1970; Fromm, 1994), la psiquiatría se transformó en una estrategia biopolítica dirigida al espacio social y legitimada para resolver gran parte de los conflictos sociales, emergiendo también con fuerza la labor preventiva que con el tiempo se le ha ido atribuyendo (Caponi, 2012).

\subsection{Objetivos}

El objetivo principal de este estudio, mediante el cual se construirán y estructurarán los demás, radica en comprender el modo en que se patologiza la conducta; a partir de qué discursos y desde qué perspectivas una conducta social, conflictiva en este caso, pasa a ser vista como una situación objeto de las ciencias de la salud. Para esto, buscaré:

- conocer las definiciones de "salud" y "enfermedad" que se manejan desde los ámbitos profesionales dedicados a esta cuestión;

- comprender a partir de qué circunstancias o cualidades se construye la identidad que se atribuye al paciente por parte del circuito asistencial; 
- conocer la definición del trastorno disocial en concreto, elaborada no solo a partir de la literatura médica, sino también mediante la práctica médica del profesional, su contacto con pacientes "de carne y hueso"; y

- averiguar de qué manera los profesionales consideran que esta circunstancia se articula con el entorno cercano del menor y con el contexto general más amplio.

En definitiva, y sin negar el conflicto sobre el que se elabora el diagnóstico, en este trabajo trataré de averiguar en qué se fundamenta y cómo se desarrolla la intervención clínica en una cuestión cuya manifestación aparece más como un conflicto comportamental de cara a la sociedad que como un conjunto de síntomas o un malestar vivido en primera persona por el paciente.

\subsection{Muestra}

Para acceder a las perspectivas que considero relevantes en este trabajo, realicé el estudio atendiendo a los discursos de los profesionales acerca de la problemática abordada y al proceso completo al que los jóvenes son sometidos, articulando los conceptos generales con las prácticas desarrolladas desde diferentes ámbitos de abordaje, en diferente proporción según las necesidades que percibí a lo largo de la investigación: la psicología, la psiquiatría y la educación social. Así, los informantes, cuyos nombres he cambiado para este trabajo por motivos de confidencialidad, fueron:

\begin{tabular}{|l|l|}
\hline Iván & Psicólogo clínico en consulta privada y orientador en un centro escolar \\
\hline Susana & Psicóloga de un hospital de día para menores \\
\hline Lucía & Psiquiatra infanto-juvenil e investigadora de un hospital público \\
\hline Pablo & Psicólogo de un centro terapéutico cerrado de menores concertado \\
\hline Sergio & Psicólogo de un centro terapéutico cerrado de menores privado \\
\hline Marcos & Psicólogo de un correccional de menores \\
\hline Paula & Psicóloga de un hospital de día para menores \\
\hline Laia & Educadora social \\
\hline
\end{tabular}




\section{Discursos expertos en torno al trastorno disocial}

\section{1 Salud y enfermedad. El contexto y la edad como marcos definitorios}

Como afirma Martínez (1998), en antropología los síntomas deben tratarse desde una perspectiva interpretativa, como expresiones que vehiculan significados en un contexto determinado. Los síntomas psíquicos son aquellas expresiones del paciente acerca de sí mismo, que solo son identificadas como tales si el observador, en primera, segunda o tercera persona, las considera de esta manera. Por ello, para comprender todo el fenómeno de patologización de la conducta, es esencial atender primero a qué es considerado "normal" y qué "patológico" desde el punto de vista de aquellos designados socialmente para gestionar el conflicto a partir de una perspectiva médica.

Fromm (1994) define la salud mental a partir de dos posibles acepciones. Por un lado, la describe como la capacidad del sujeto para desempeñar el rol social que le ha sido asignado sin perturbar su entorno social. Por otro, propone una acepción estadística, que refiere a la "normalidad", a la adecuación del individuo a las conductas o emociones más frecuentes en su grupo. Caponi (2012) propone una concepción de la normalidad también estadística, además de otra relacionada con el "deber ser", con el modo en el que el grupo espera que el individuo se desenvuelva en sus circunstancias; con cómo desempeñe su rol social, al fin y al cabo.

El trastorno sería, entonces, el fracaso respecto al ideal de funcionamiento de un individuo (Arasanz, 2016), que viene determinado por sus características personales. En relación a la infancia y la adolescencia, atendemos a la tipificación, formal o informal, de una serie de conductas que, si bien pueden llegar a ser conflictivas, no son consideradas patológicas debido al reconocimiento de estas etapas de vida como de necesaria confrontación (Monseny, 2016). Así, los comportamientos patológicos serían aquellos que causaran más conflicto de lo esperable. De esta manera, el rol del niño y adolescente llevaría implícita cierta confrontación, que es considerada como estadísticamente normal mientras no supere unos niveles estipulados.

Así lo señalaba Paula, afirmando que los casos en los que los niños no provocaban ningún tipo de conflicto ni enfrentamiento en casa eran aquellos en los que, con el tiempo, surgiría una patología mayor. En su trabajo como orienta- 
dor de una institución escolar, Iván consideraba difícil distinguir las conductas normales, propias del desarrollo, de las patológicas en el periodo de la adolescencia. Sergio señalaba algo similar a Paula en el caso de los adolescentes, de quienes interpreta ciertas actitudes como la creación de una identidad propia.

Las consultas previas que todos los informantes han manifestado requerir con los pacientes, aun habiendo sido estos previamente diagnosticados por otra institución, dan cuenta de la dificultad para determinar qué puede considerarse patológico y qué no a la hora de establecer un dictamen. Así, Marcos afirma: "Muchos vienen diagnosticados y nosotros lo que hacemos es retirar el diagnóstico". Considera complicado el dictamen en la población adolescente, más aún en trastornos cuyos criterios se basan casi únicamente en rasgos conductuales, por lo que solo consideran la presencia del TD en los casos en los que existe comorbilidad con algún otro trastorno. Para él, los criterios estipulados no bastarían para determinar la presencia de esta patología por sí solos; sería así una suerte de trastorno complemento de otros, nunca una patología por sí solo.

También Pablo, desde su experiencia en una institución de internamiento 24 horas, afirma que según los criterios conductuales prácticamente la totalidad de los jóvenes con los que trata podría haber recibido este diagnóstico, sin que esto llegase a suceder. En este caso, lo determinante parecía el modo de acceso de los menores al recurso asistencial, en función de su trayectoria, sin que la diferencia entre haber sido diagnosticado o no fuera realmente relevante en la práctica cotidiana. Así, aunque de otra manera, en este caso también parece que el TD pierde peso como entidad propia y condicionante en la estrategia a seguir.

Este condicionamiento del contexto, que observaremos en un apartado posterior, aparece en todos los discursos como un factor determinante en múltiples dimensiones. En lo que nos atañe ahora, cabe destacar que, como afirma Herrera (2016), las reglas, la norma, están limitadas a un marco; son dadas en un momento concreto para un proyecto determinado. Esto lo reflejaba Lucía, afirmando que un mismo conjunto de síntomas podía ser una enfermedad o no en función del contexto. Para ella, el cuadro debía considerarse un trastorno cuando causaba sufrimiento, sin llegar a determinar si debía ser el propio paciente el que lo percibiera de esa manera, su entorno o ambos.

Por otro lado, atendiendo a los criterios necesarios para considerar que el paciente se había curado, en correspondencia con las características del tipo 
de patologización, los informantes se refirieron a cambios relativos al modo de actuación: la asunción de responsabilidades, el cumplimiento de normas, el respeto a la propiedad privada ajena, la expresión adecuada de emociones... Esto, una vez más, da cuenta del TD como una patología "de paciente para fuera", sensible para él en la medida en que condiciona su relación con el entorno, pero no tanto perceptible por las emociones que de por sí puede causarle.

En definitiva, con el trastorno disocial atendemos a un caso de patología construida meramente a partir de criterios conductuales y referidos al entorno social, para la que no existe un criterio uniforme de diagnóstico más allá de las posibilidades de aplicación de los manuales oficiales (DSM y CIE). Además del peso que los informantes atribuyen al contexto en el que la patología se desarrolla, vemos cómo también el entorno asistencial condiciona la propia visión de los profesionales, llegando a relativizarse su importancia como diagnóstico individual en los entornos en los que la conflictividad es inherente a la institución. Donde las conductas estipuladas se han convertido en la normalidad estadística, el TD requiere complementarse con otros para ser reconocido como tal.

\subsection{Trastorno disocial: de la conducta divergente a la patología}

Ante la ya mencionada falta de una base biológica demostrada en los trastornos mentales, para elaborar un diagnóstico los profesionales deben servirse de una serie de observaciones y la identificación y clasificación de conductas. Como hemos visto, si bien en medicina lo habitual es considerar que una persona está físicamente enferma cuando su organismo presenta un funcionamiento fuera de lo normal, cuando tiene disease identificable, en el ámbito de la salud mental se considera que hay patología cuando su conducta va en contra de determinadas normas éticas, políticas y sociales (Szasz, 1970).

En el caso del TD, por un lado, la duración en el tiempo del conflicto aparecía como un criterio diagnóstico importante: no se considerarían como síntomas conductas aisladas. También era importante la «gravedad», la profundidad atribuida al conjunto global de síntomas, que condicionaba la inclusión de los pacientes a los distintos dispositivos asistenciales o su derivación a otros. En 
relación a la vivencia subjetiva de los pacientes respecto a su situación, los informantes no referían tanto la vivencia de los pacientes como la consideración del profesional respecto a su poca adecuación a la "realidad" (Susana y Pablo).

Las conductas patologizadas más frecuentes en los discursos de los informantes eran la impulsividad, la violencia y la interpretación "errónea" de la realidad. Iván consideraba que la impulsividad traía consigo una falta de intencionalidad explícita que eximía en cierta medida al niño de responsabilidad. Ante esto, en primer lugar, se buscaba fomentar la reflexión previa a la actuación y la capacidad de reparación y de asunción de las consecuencias de los propios actos (Susana y Pablo). El síntoma sin duda más referido es la agresividad, principalmente física. Esto implicaba la imposibilidad de integrar a muchos pacientes en los centros escolares mayoritarios (Susana, Pablo y Paula), la limitación del número de pacientes con TD en los grupos de terapia (Paula), la imposición de medidas judiciales basadas en la libertad vigilada (Laia) o el internamiento en centros de reforma (Marcos).

Respecto a las causas del trastorno disocial, la mayoría de informantes coinciden en señalar una etiología biopsicosocial. Sin embargo, el peso que dan a estos tres factores difiere. Lucía, con profesión psiquiátrica, dio gran peso al factor biológico en la entrevista; Pablo y Paula simplemente mencionaron una predisposición genética a recurrir a determinadas conductas; Iván dudó si esta inclinación existe o no; Susana y Sergio simplemente mencionaron el trío de factores. Marcos ni siquiera mencionó la biología. Además, Paula cuestionaba si las anomalías biológicas que las investigaciones podían descubrir no serían más bien una cristalización, el reflejo biológico, de una situación cultural y social.

En el discurso psicológico el protagonismo lo adquiría casi por completo, más allá de las menciones eventuales a la predisposición de las que ya he dado cuenta, los factores sociales y su efecto en la psicología individual, en las estrategias de expresión y en la relación con las figuras de autoridad y grupo de pares ("Habían aprendido que la violencia era eficaz y se habían vuelto expertos en violencia para llegar a donde ellos querían", señala Pablo). Así, el TD para ellos y ellas sería predominantemente un trastorno social, tanto por su origen como por su efecto. 


\subsection{El paciente como menor, expresión y víctima}

Respecto a los menores diagnosticados, hay dos factores principales que condicionan la identidad que el sistema les atribuye: la edad y la patología. Como hemos visto, el hecho de encontrarse en un periodo de la vida identificado en nuestra cultura como conflictivo de por sí condiciona la intensidad de enfrentamiento socialmente aceptada como "normal". Pero además, esta dimensión etaria se manifiesta en la atribución de capacidades o potencialidad sobre la población referida. La idea del niño concebido como un ser «aún por terminar» legitima las intervenciones educativas que estructuran su vida de manera más o menos estricta y el tratamiento temprano como modo de evitar un desarrollo del trastorno antisocial de la personalidad.

La situación legal de menor del paciente, condicionada también por la edad, implica además una acotación a su capacidad de decisión. Si bien en los casos en los que los menores son sometidos a medidas asistenciales por decreto judicial esta dimensión no parece condicionada tanto por la edad como por el circuito penal, en los recursos en los que es la familia la que decide el acceso del menor queda de manifiesto cómo la edad puede determinar por completo el desarrollo del tratamiento, quedando supeditada la voluntad del paciente a la consecución de lo que se concibe como un bien mayor.

Sin embargo, a pesar de esta condición de subordinación aparentemente inherente al niño o adolescente, en general se le reconoce capacidad de agencia, deseable o no, dentro de los márgenes que las instituciones le imponen. Pablo, por ejemplo, relata su habilidad para "medir" a los adultos y actuar en consecuencia de la manera que, desde su perspectiva, les beneficie más. Laia, por su parte, alude a la imposibilidad de obligar a una persona a beneficiarse de un tratamiento psicológico en contra de su voluntad.

Aludiendo a la experiencia subjetiva del paciente, tanto Iván como Sergio afirman que estas poblaciones, aunque a menudo aparecen como maltratadoras, también han podido ser o son susceptibles de llegar a ser víctimas de maltrato en cualquier ámbito de su vida. Así, el menor no aparece solo como ejecutor de violencia, también constituye un individuo en riesgo. Respecto a la responsabilidad percibida en el conflicto, todos los informantes dan cuenta de 
la variabilidad que presenta el fenómeno: mientras la mayoría reconoce formar parte de un enfrentamiento, algunos se consideran víctimas y otros, culpables, en diferente medida.

Sin embargo, el reconocimiento de la importancia del factor ambiental supone de por sí una llamada de atención sobre la necesidad de atender también a actores sociales más allá del propio paciente. En esta línea, Paula señala la posibilidad de ver al niño como síntoma de una dinámica familiar patológica, donde este actuaría como el "paciente designado", la expresión de un problema mayor, no el problema en sí. De esta manera, toda la familia sería considerada patológica, resultando el menor el miembro expresivo.

En lo referente a la dimensión patológica de su identidad, los profesionales a los que tuve acceso, por trabajar precisamente este aspecto de su vida, perciben a los menores con este diagnóstico en gran parte en función de la patología que se les ha achacado, siendo consecuentes en sus estrategias con las características que a ello se atribuyen. Sin embargo, todos ellos se muestran explícitamente reacios a favorecer el desarrollo de etiquetas, buscando evitar por un lado la estigmatización y, por otro, la instrumentalización del menor de la patología para justificar sus actos y la estructuración de su identidad a partir de la experiencia terapéutica, a menudo totalizadora.

\subsection{Condicionamientos legales y administrativos al proceso terapéutico}

Como ya hemos visto, una de las dimensiones en que la legislación condiciona la gestión de esta cuestión es a partir del estatus de los pacientes de menores de edad y, por tanto, sometidos a la voluntad de sus tutores legales, sean estos los padres, otros miembros de la familia o entorno cercano o la Administración. Sin embargo, la experiencia de los informantes ha puesto de manifiesto los diferentes modos en que las instituciones y su gestión influyen sobre la trayectoria y la experiencia de los usuarios en el circuito asistencial.

Desde la misma base del proceso, Iván da cuenta de los casos que él mismo como orientador puede diagnosticar y qué casos debe remitir a la Administración para que sea un psiquiatra autorizado quien confirme el diagnóstico, entre los que se encontrarían los menores con trastorno disocial. A los hospitales de día, los menores pueden ser remitidos por psiquiatras de la Comunidad de Ma- 
drid o por iniciativa familiar. Sin embargo, si se da el primer caso, la negativa de la familia implicaría su sometimiento a una revisión por parte de las autoridades que podría degenerar en la retirada de la patria potestad. Incluso, tras el ingreso del menor, los ayuntamientos tienen un servicio social de seguimiento de los casos, según relata Susana. Esta pérdida de la custodia del menor, según cuenta Pablo, era habitual en los casos que él trató en el centro terapéutico en el que trabajó.

Por tratarse de una patología donde las transgresiones de la ley son habituales, se aprecia también una relación relativamente frecuente con las instituciones jurídico-penales, que condicionan el proceso en dos sentidos. Por un lado, la imposición del juez del recurso social al que el menor debe acudir, que elimina no solo la capacidad de decisión del menor, sino también de su familia. Por otro, la constricción temporal que suponen en el proceso terapéutico tanto el tiempo transcurrido entre el delito y el juicio, que implica un castigo tardío (Laia y Pablo), como el plazo establecido desde el juzgado para el alta obligatoria del paciente, que a veces implica el abandono prematuro del tratamiento (Pablo, Marcos y Laia).

También la provisión de recursos económicos, financiación, para el tratamiento integral de estos casos es denunciada como una de las carencias principales del circuito asistencial. Tanto Laia como Pablo señalan lo perjudicial de la alta rotación de personal, así como la imposibilidad de realizar el trabajo global con las familias que sería necesario en estos casos, ambos problemas provocados por la falta de presupuesto.

Así, las instituciones administrativas condicionan el proceso terapéutico en tres sentidos: por su calidad de menores de edad, el gobierno de las vidas de los pacientes es sometido a un escrutinio mayor, llegando en ocasiones a cambiar de «manos» la responsabilidad de sus condiciones; la estrecha relación de sus estrategias conductuales con la transgresión legal los pone a menudo en contacto con el circuito penal, que condiciona tanto su ingreso en las instituciones como su alta, a veces más por criterios legales que sanitarios; y por último, por situarse muchos de los recursos entre las posibilidades provistas por la sanidad pública, la calidad del servicio y la integralidad del tratamiento se encuentran sometidas a la capacidad económica del sistema, a menudo insuficiente. 


\subsection{Responsabilización del desarrollo del TD y la importancia del contexto}

La mencionada definición del paciente como un síntoma de una dinámica familiar patológica llama la atención sobre la responsabilidad que desde el ámbito asistencial se atribuye al grupo familiar. Aunque también se señalaba la incapacidad en muchos casos por parte de la Administración para cubrir las necesidades de los menores de manera que la patología no llegase a ser tal, este grupo y los procesos de socialización que se ejercen en su seno fueron los principales aludidos en todas las entrevistas. Se señala una "mala" socialización, atribuida a diferentes circunstancias o procesos, entre los que las explicaciones relativas a dificultades atribuidas a características propias de los padres fueron minoritarias en comparación con aquellas que aludían a la implicación de los parientes en la socialización, su grado y su estructuración. En este sentido, la característica más señalada como dinámica patológica fue la falta de estructura unificada en la educación del menor, a menudo causada por el divorcio de los progenitores: "A veces hay tal desorganización familiar que es imposible que un chaval se organice internamente [... . . Como que reciben mensajes contradictorios todo el tiempo" (Susana).

Esta falta de organización, estrategia educativa, según ellos puede derivar en una confusión de roles, ya sea entre los propios padres o entre padres e hijos (Pablo, Sergio y Paula): "No sé, casas en las que se ven cosas que no se tienen que ver, casas en las que no están bien definidos los roles de padres, hijos, hermanos y demás, casos en los que los propios padres rompen reglas" (Pablo). También se ha señalado una falta de atención por parte de los padres a las necesidades de los menores: Susana alude al abandono afectivo; Sergio alude, entre otras cosas, a la hiperexigencia académica en detrimento de las necesidades afectivas de los hijos. Por esto, desde la mayoría de los recursos se llama la atención sobre la necesidad de implicar en el proceso terapéutico a las familias, tratando de propiciarlo en la medida de sus posibilidades.

Más allá de la familia, el grupo de pares también adquiere cierta relevancia tanto en la génesis del problema como en la solución, condicionando de manera evidente la experiencia del ocio y las rutinas, así como el tipo de relaciones que el menor aprendía a establecer y las estrategias que desarrollaba para mantener 
una posición social satisfactoria. En relación con esto, menos Iván, todos los informantes consideraron que a menudo es necesario cambiar de entorno si este no se puede transformar hacia uno más beneficioso para el individuo. Marcos, Sergio y Pablo señalan respecto al internamiento del menor que llegar a un entorno estructurado facilita la normalización, además de separar al menor de las dinámicas consideradas como tóxicas. Para ellos, la imposición de patrones normalizadores reestructura al sujeto, adecuándolo al objetivo establecido. Sin embargo, ninguno recomienda una atomización del individuo: todos refieren los beneficios del trabajo en grupo, al menos para determinadas actividades.

Respecto a causas o dinámicas estructurales, tanto Iván como Paula se refirieron a modas a la hora de emitir diagnósticos: mientras Paula parece atribuirlo a dinámicas sociales que provocan malestares concretos, Iván señala más bien a una moda "en el ojo" de quien diagnostica, a una focalización de la visión experta en patologías concretas que provoca una mayor laxitud a la hora de determinar la presencia de patología, multiplicándose los casos resultantes. En relación a la situación económica general, Marcos señala que con las crisis las conductas disruptivas disminuyen, considerando Paula que con las crisis económicas las patologías en general aumentan, ya que el malestar de los padres crece y repercute en sus hijos.

En definitiva, en lo que respecta al contexto social y cultural que los profesionales consideran a la hora de explicar y tratar el TD, la familia adquiere un protagonismo evidente. Se la caracteriza como desestructurada, generalmente a causa de conflicto entre los padres o falta de comunicación y acuerdo, además de poco ajustado a las necesidades del niño o la niña. A pesar del papel que juega en la socialización de los adolescentes, el grupo de pares pocas veces aparece en los discursos a la hora de tratar el origen de la patología, aunque sí lo hace en el tratamiento, en forma de grupo de terapia. Respecto al contexto socioeconómico general, tiene más presencia en forma de limitaciones administrativas, referidas en el apartado anterior, que de condiciones sociales estructurales. Así, se representa a menudo a las familias como unidades colectivas relativamente autónomas del grupo amplio y de las dinámicas sociales en los que están insertas. 


\section{Conclusiones}

Como hemos observado, al complementar la lectura de literatura médica con los discursos de estos profesionales queda de manifiesto cómo no se aplican de la misma manera los criterios diagnósticos en todos los estamentos del circuito asistencial. En ocasiones son relativizados en función del contexto, evidenciándose cierta falta de acuerdo en relación a las conductas que son consideradas patológicas. Aunque la etiología de la patología era considerada por la mayoría de informantes como biopsicosocial, se han manifestado también ciertas diferencias en cuanto al peso de cada uno de los factores, siendo la diferencia sustancial entre la perspectiva psiquiátrica, más biologicista, y la psicológica, más centrada en la trayectoria del paciente y su relación con el contexto socializador. Aun así, en lo que respecta al peso de los diferentes actores que constituyen la dimensión social, la familia se ha presentado indudablemente como protagonista y determinante.

Se establece así una suerte de triángulo de relación entre el paciente; su grupo cercano, la familia; y las instituciones, representadas por los expertos médicos y educativos y la Administración pública. Resulta evidente la asimetría de poder entre agentes: las instituciones marcan el "deber hacer", el modo en que se debe enfrentar la cuestión, ostentado así la capacidad de definir la realidad a la que el conjunto de actores se enfrenta. Aunque el paciente se muestra formalmente sometido a la autoridad familiar o institucional, se le reconoce cierta capacidad de actuación autónoma, determinante a la hora de someterse o no a las premisas impuestas desde su exterior, determinando la consideración de éxito o fracaso en el tratamiento. Por su parte, si bien en las primeras instancias del proceso asistencial la familia tiene la capacidad de decisión esencial, pronto las instituciones se apropian de ella mediante la legitimación médica, por un lado, y las medidas legales de control del menor, por otro, quedando este grupo como mero agente colaborador en la "reeducación" del menor en algunos casos y como objetivo de la misma en otros.

$\mathrm{Al}$ atender al agente institucional, por su carácter colectivo, debemos tener presente que no se trata de un grupo homogéneo en cuanto a perspectivas y la misma capacidad de incidencia o capacidad de actuación. En este sentido, cabe recordar lo que Young (1993) llamó código institucional: aquel que somete tanto al paciente como al profesional a la autoridad del régimen terapéutico. A pesar 
de ser los informantes representantes del saber experto, sus experiencias relataban la incapacidad de tomar decisiones individuales, la obligación de hacerlo en función de las pautas establecidas para su centro o disciplina. Más allá de este código, incluso, es destacable la fuerte influencia que de diferentes formas ejerce la Administración sobre los procesos médicos.

A pesar de la doble condición de subordinación de estos jóvenes, en calidad de menores de edad y de enfermos mentales, la práctica profesional atiende también a otros factores determinantes en el conflicto, sin culpabilizar siempre al individuo. El análisis, al no dejar de lado el entorno cercano en el que se desarrollan las conductas disruptivas, permite una visión del sujeto como resultado de un proceso extenso en el tiempo, poniendo la atención no solo en el conflicto presente, sino también en la trayectoria individual desde un punto de vista relacional.

Respecto a la terapia, el peso que se da al trabajo en grupo en la mayoría de recursos según la experiencia de los informantes denota también el reconocimiento de la patología como una entidad eminentemente social. "El grupo es terapéutico", afirmaba Paula. Bajo esta misma lógica, cabe señalar que las referencias a la conducta de los menores y a la curación al conseguir adaptar su conducta a los patrones estipulados, dotándolo de recursos expresivos y de gestión de las emociones, sigue presentando una patología desde el exterior, donde las emociones y vivencia del paciente pasan a un segundo plano, quedando supeditadas a los objetivos conductuales y, aparentemente, dándose por hecho que se tornarán positivas con su adecuación a la norma. 


\section{Bibliografía}

Aguirre, C. (2007). "Hegemonía”. En Szurmuk, M. y McKeeIrwin, R. (eds.). Diccionario de Estudios Culturales Latinoamericanos. México: Siglo XXI Editores.

American Psychiatric Association. DSM-IV. Manual diagnóstico y estadístico de los trastornos mentales. [Online <http://www.psicomed.net/dsmiv/dsmiv1.html\#f91.3> ]

Arasanz, M. D. (2016). "El Trastorno de Conducta, su sentido”. En MonSENy Bonifasi, J. (coord.). ¿Trastornos de conducta o conductas que trastornan? Los trastornos de conducta en los niños y adolescentes. Barcelona: Horsori Editorial.

CAponi, S. (2012). Loucos e degenerados: uma genealogia da psiquiatría ampliada. Rio de Janeiro: Editora Fiocruz.

Cova, F; Pérez-Salas, C. P.; Parada, B. V; Saldivia, S.; Rioseco, P. y Soto, O. (2012). "Inteligencia y conductas delictuales en Adolescentes Chilenos". Revista Latinoamericana de Ciencias Sociales, Niñez y Juventud, $10(1) \div 441-452$.

Delgado, M.; González, A.; Moreno, A.; Pérez, E.; Ponte, N. y Sierra, A. C. (1994). ¿Locos o malos? Una revisión del trastorno de personalidad antisocial o disocial”. Revista de la Asociación Española de Neuropsiquiatría, vol. XIV, n. ${ }^{\circ}$ 51, pp. 611-632.

Dios de Vega, J. L. (2002). Trastornos disociales en la adolescencia. Estudio de su incidencia en la Comunidad de Madrid. Madrid: Defensor del Menor en la Comunidad de Madrid.

Dueñas, G. (2007). “'Nuevos dispositivos de control de conducta?”. Revista Novedades Educativas, n. 196.

Fassin, D. (1999). "La patetización del mundo. Ensayo de antropología política del sufrimiento”. En Viveros Viguya, M. y Garay Ariza, G. H. (comp.). Cuerpo, diferencias y desigualdades. Santa Fe de Bogotá: Centro de Estudios Sociales.

- (2010). "El irresistible ascenso del derecho a la vida. Razón humanitaria y justicia social”. Revista de Antropología Social, 19: 191-204.

Ferreras, C. (2016). "Més consultes per trastorns en adolescents". En La Vanguardia. 28 de enero de 2016. 
Font, E. y Vidal, L. (2016). "Los caminos de la pedagogía: vínculo, contención, consentimiento". En Monseny Bonifasi, J. (coord). ¿'Trastornos de conducta o conductas que trastornan? Los trastornos de conducta en los niños y adolescentes. Barcelona: Horsori Editorial.

Foucault, M. (1998). Historia de la sexualidad I. La voluntad de saber. Madrid: Siglo XXI Editores.

- (2007). El poder psiquiátrico. Curso en el Collège de France (1973-1974). Buenos Aires: Fondo de Cultura Económica.

Fromm, E. (1994). La patología de la normalidad. Barcelona: Paidós.

González Pardo, H. y Pérez Álvarez, M. (2007). La invención de los trastornos mentales. ¿Escuchando al fármaco o al paciente? Madrid: Alianza Editorial.

Good, B. J. (2003). Medicina, racionalidad y experiencia. Una perspectiva antropológica. Barcelona: Edicions Bellaterra.

Guijarro, R. (2016). "El malestar de unos y otros. Asesoramiento psicopedagógico y respuesta educativa”. En Monseny Bonifasi, J. (coord). $¿$ Trastornos de conducta o conductas que trastornan? Los trastornos de conducta en los niños y adolescentes. Barcelona: Horsori Editorial.

Herrera, M. (2016). "Cuando lo que se trastorna es la función educativa (Una aportación desde el SEETDIC)”. En Monseny Bonifasi, J. (coord). $¿$ Trastornos de conducta o conductas que trastornan? Los trastornos de conducta en los niños y adolescentes. Barcelona: Horsori Editorial.

Lorenzini, D. (2010). "Para acabar con la verdad-demostración. Bachelard, Canguilhem, Foucault y la historia de los «regímenes de verdad»". Revista Laguna, 26, marzo 2010, pp. 9-34.

Kleinman, A. (1991). Rethinking Psychiatry. From cultural category to personal experience. New York: The Free Press.

Matalí, J. (coord.) (2016). Adolescents amb trastorns de comportament. Compodem detectar-los? Què cal fer? Barcelona: Hospital Sant Joan de Déu (ed.).

Martínez, A. (2016). "Los trastornos de conducta, un cajón de sastre". En Monseny Bonifasi, J. (coord). ¿Trastornos de conducta o conductas que trastornan? Los trastornos de conducta en los niños y adolescentes. Barcelona: Horsori Editorial.

Martínez Hernáez, Á. (1998). ¿Has visto cómo llora un cerezo? Barcelona: Publicacions de la Universitat de Barcelona. 
Martínez Hernáez, Á. (2008). Antropología médica. Teorias sobre la cultura, el poder y la enfermedad. Barcelona: Anthropos.

Medel, E. (2016). Infancias contemporáneas. Retos educativos. Barcelona: Editorial OUC.

Mobilli Rojas, A. y Rojas Malpica, C. (2006)."Aproximación al adolescente con trastorno de conducta disocial". Artemisa medigraphic, col. VIII, n. ${ }^{\circ}$ 2. Agosto 2006.

Moffitt, T. E. (1993). "Adolescence-limited and life-course-persisten antisocial behavior: a developmental taxonomy". Psychological Review, vol. 100, n. $^{\circ}$, pp. 674-701.

Monseny Bonifasi, J. (2016). "La conducta habla”. En Monseny Bonifasi, $\mathrm{J}$. (coord). ¿Trastornos de conducta o conductas que trastornan? Los trastornos de conducta en los niños y adolescentes. Barcelona: Horsori Editorial.

Organización Mundial de la Salud. CIE-10. Clasificación Internacional de Enfermedades. [Online <http://www.psicomed.net/cie_10/cie10_F91. html\#f91.8> ]

Pajuelo, L. (2016). "El Trastorno de Conducta en un aula ordinaria”. En Monseny Bonifasi, J. (coord). ¿Trastornos de conducta o conductas que trastornan? Los trastornos de conducta en los niños y adolescentes. Barcelona: Horsori Editorial.

Pineda, D. A. y Puerta, I. C. (2001). Prevalencia estimada del Trastorno Disocial de la Conducta en adolescentes colombianos. [Online <http://www. psiquiatria.com/psiq_general_y_otras_areas/epidemiologia-78/prevalencia-estimada-del-trastorno-disocial-de-la-conducta-en-adolescentes-colombianos/> ]

Romero, E. (2001) “El constructo psicopatía en la infancia y la adolescencia: del trastorno de conducta a la personalidad antisocial". Anuario de Psicología, vol. 32, n. 3 , pp. 25-49.

Ruvalcaba, N.; Salazar, E. y Gallegos, J. (2012). "Competencias socioemocionales y variables sociodemográficas asociadas a conductas disociales en adolescentes mexicanos". Revista CES Psicología, 5 (1), 1-10.

Szasz, T. S. (1970). Ideología y enfermedad mental. Buenos Aires: Amorrortu editores.

- (2008). El mito de la enfermedad mental. Bases para una teoría de la conducta personal. [ $3^{\mathrm{a}} \mathrm{Ed}$.]. Buenos Aires: Amorrortu editores. 
Soutullo Esperón, C.; y Mardomingo Sanz, M. J. (coords.) (2010). Manual de Psiquiatría del Niño y del Adolescente. Asociación Española de Psiquiatría del Niño y del Adolescente (AEPNYA). Madrid: Editorial Médica Panamericana.

Toro, J. (1999). Psiquiatría de la adolescencia. Madrid: Aula Médica Ediciones. VÁsquez Rocca, A. (2011)."Antipsiquiatría. Deconstrucción del concepto de enfermedad mental y crítica de la «razón psiquiátrica”». Nómadas. Revista Crítica de Ciencias Sociales y Jurídicas, 31 (2011, 3).

Young, A. (1982). “The Anthropology of Illness and Sickness”. Annual Review of Anthropology, 11, pp. 175-181.

- (1993). "A Description of How Ideology Shapes Knowledge of a Mental Disorder (Posttraumatic Stress Disorder)". En Lindenbaum, S. y Lock, M. M. (eds.). Knowledge, power and practice. University of California Press.

\section{Recursos audiovisuales}

Instituto de Capacitación Parlamentaria (2015). Jornada Biomedicalización y Patologización de los Malestares Infantiles Actuales. PARTE 1. Publicado el 26 de agosto de 2015. [Online <https://youtu.be/cxeWTS$8 y 02 Q>]$ 